\title{
Search for GRB neutrino emission according to the photospheric model with the ANTARES telescope
}

\author{
Matteo Sanguineti on the behalf of the ANTARES Collaboration* \\ Università degli Studi di Genova, INFN Genova
}

E-mail: matteo.sanguineti@ge.infn.it

\begin{abstract}
The ANTARES detector is the largest neutrino telescope currently in operation in the North Hemisphere. One of the main goals of the ANTARES detector is the search for cosmic neutrino sources including transient sources like GRBs. In the so-called photospheric model for the emission from GRBs the interaction of the radiation field with the leptonic component of the outflow could lower the expected energy spectrum of the associated neutrino emission from GRBs. In coincidence with a GRB alert from a satellite, ANTARES stores a window of few minutes of unfiltered data. A dedicated directional filtering and reconstruction is applied offline to enhance the sensitivity in the lower energy range of the ANTARES detector $(50 \mathrm{GeV}-10 \mathrm{TeV})$. The expected improvement as derived from Monte Carlo simulations will be presented.
\end{abstract}

The 34th International Cosmic Ray Conference,

30 July- 6 August, 2015

The Hague, The Netherlands

* Speaker. 


\section{Introduction}

Gamma ray bursts (GRB) belong to one of the most energetic phenomena of the Universe, but their origin remained a mystery for many years. A milestone in the GRB detection was the launch of the Compton Gamma-Ray Observatory, in particular the Burst and Transient Experiment (BATSE) detected over 2700 bursts [1]. BATSE showed that GRBs are distributed isotropically in the sky without any dipole or quadrupole moments, indicating an extragalactic origin later confirmed by redshift measurement [2].

The fireball shock model is the best-known scenario that has been put forth to explain the gamma ray emission mechanism associated with GRBs. It predicts that different shock waves will be traveling at different relativistic speeds, and it is the interaction between these different shock fronts that cause the energetic gamma-ray emissions.

A new widely discussed scenario is the photospheric model [3][4], which predicts a neutrino emission nearer to the central engine where the relativistic jet is still optically thick. This model is interesting because it predicts some features of the gamma ray spectrum of GRBs that are not foreseen by the fireball scenario, like the Amati correlation [5]. The photospheric model predicts a lower energetic neutrino spectrum respect to internal shock model and it has already been investigated by the IceCube collaboration [6]. The fireball model has already been tested by different ANTARES analyses [7] [8]. We will focus on photospheric model, so we will exploit a special data set that could offer a better sensitivity in the lower energy range. Also a low-energy optimized reconstruction algorithm and a directional filter have been used to additionally improve the sensitivity in the interesting energy range.

\section{The photospheric model}

Like in the fireball model the presence of a jet-like relativistic outflow is assumed. The photospheric model predicts the conversion of a fraction of the bulk kinetic energy into radiation energy through a dissipation mechanism in the neighbourhood of the photosphere. The photosphere occurs in the acceleration phase $r<r_{\text {sat }}$ if the outflow is magnetically dominated, on the other hand in the barionic case the photosphere occurs in the coasting phase $\left(r>r_{\text {sat }}\right)$, where $r_{\text {sat }}$ is the saturation radius [3].

In the barionic case, two different mechanisms can lead to dissipation: the dissipation of magnetohydrodynamic (MHD) turbulence or semi-relativistic shocks [9] with Lorentz factor $\Gamma_{r} \sim 1$ as in internal shocks. In the magnetically dominated case, before the dissipation occurs the total jet luminosity consists of a toroidal magnetic field component and a proton bulk kinetic energy component [3]. Calculations and simulations of such baryonic and magnetic dissipative photospheres predict a spectrum similar to the observed characteristic "Band" spectrum [10], parameterized as

$$
\frac{d N_{\gamma}}{d E} \propto\left(\frac{E}{E_{b r}}\right)^{x_{p h}}
$$

where a burst with z=2 redshift shows $E_{b r}$ around $300 \mathrm{keV}$ and

$$
\begin{aligned}
& -x_{p h}=-1 \text { for } E>E_{b r} \\
& \text { - } x_{p h}=-2 \text { for } E>E_{b r}
\end{aligned}
$$


In the barionic photosphere scenario protons and electrons are assumed to be accelerated through a Fermi-first order acceleration mechanism in the surrounding magnetic fields. A similar process is also expected in the magnetic photosphere scenario.

Neutrinos are mainly produced though charged pion and kaon decays; these charged mesons come from $\mathrm{p} \gamma$ and pp interactions. For energies below $1 \mathrm{GeV}$ the cross section is dominated by resonances while at higher energies multi-pion production prevails.

The pions decay is fully understood and neutrinos are produced mainly from this channel:

$$
\pi^{-} \rightarrow \mu^{-}+\overline{v_{\mu}} \rightarrow \overline{v_{\mu}}+e^{-}+\overline{v_{e}}+v_{\mu}
$$

and the charge conjugate particles for the $\pi^{+}$.

High-energy pions lose most of their energy through synchrotron radiation. For muons with their longer mean lifetime and smaller mass, synchrotron cooling is more severe than that of charged pions.

As previously mentioned the photospheric model can predict two different scenarios: the barionic dominated jets or magnetic fields dominated jets. These two possibilities lead to different macroscopic acceleration rates, different proper densities in the jet rest-frame, and imply a different role for magnetic dissipation in the process of particle acceleration.

In Fig. 1 two different estimates of the neutrino flux from a GRB according to the photospheric model are shown.
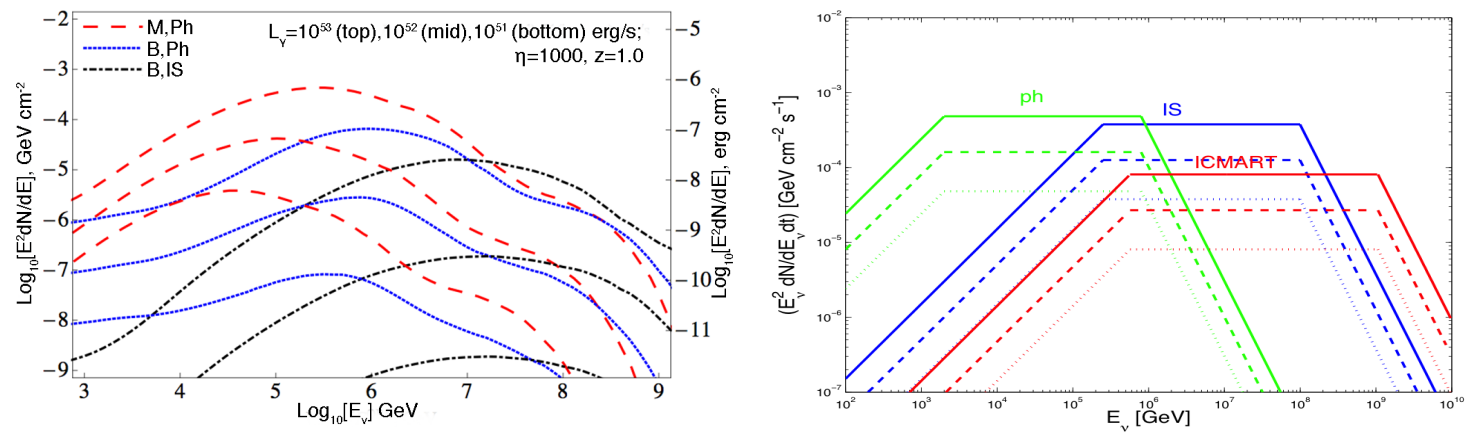

Figure 1: Left: Neutrino fluence from a single GRB assuming different dissipation models. Red, dashed: magnetic photosphere; blue, dotted: baryonic photosphere; Dot-dash: baryonic internal shock [3]. Model parameters: luminosity $L_{\gamma}=10^{53}$ (top curve), $10^{52}$ (mid curve), $10^{51}$ (bottom curve), redshift $\mathrm{z}=1$. Right: Neutrino flux from a single GRB assuming different dissipation models: "ph" (green): dissipative photosphere model; "IS" (blue): internal shock model; "ICMART" (red): internal-collision-induced magnetic reconnection and turbulence model. Model parameters: normalized luminosity $L_{\gamma, 52}=1$, variability time scale observed in the GRB light curve $\delta \mathrm{t}=0.1 \mathrm{~s}$, redshift $\mathrm{z}=1$, Lorentz factor $\gamma=250$ [4]. Three values of the ratio between photon luminosity and non-thermal proton luminosity are adopted: 0.1 (solid), 0.3 (dashed), and 1 (dotted).

The photospheric model predicts a neutrino flux at lower energies than expected from the internal shock model.

In the next section we will describe the tools which we will exploit to increase the ANTARES sensitivity in the energy range between $50 \mathrm{GeV}$ and $10 \mathrm{TeV}$ to to address the possible neutrino 
flux at lower energies. Other GRB analyses have been performed previously by ANTARES [7][8] and IceCube [11][6]. In particular the features regarding the optimization of the model discovery potential (MDP) used in this analysis have been developed and applied in [7] on the ANTARES data from end of 2007 to end of 2011. We will focus first on a generic burst with a neutrino flux $\left(E_{v}^{2} d N / d E_{v} d t\right)$ of $5 \cdot 10^{-4} \mathrm{GeV} \mathrm{cm}^{-2} \mathrm{~s}^{-1}$ and cut-offs at $2 \cdot 10^{3} \mathrm{GeV}$ and $8 \cdot 10^{5} \mathrm{GeV}$ with the future goal to study two promising candidates (GRB110918A and GRB130427A).

\section{Data sample}

For follow-up observations of bursts it is important to receive an alert in coincidence with a GRBs; for this purpose a global alert network has been created. The alert is distributed to many telescopes around the world when one of the satellites of the network detects a GRB. All satellites capable of GRB detection that were launched since BATSE (1991) are part of this network. All interested telescopes can subscribe to the system to be updated promptly with the most recent GRB information. ANTARES subscribed to the alert system, even if it is not a follow-up telescope, because we want to keep all raw data around the alert. In Fig.2 the delay is shown between the detection of a GRB by the satellite and the time of the alert message distributed, in $90 \%$ of the cases the delay is below $200 \mathrm{~s}$.

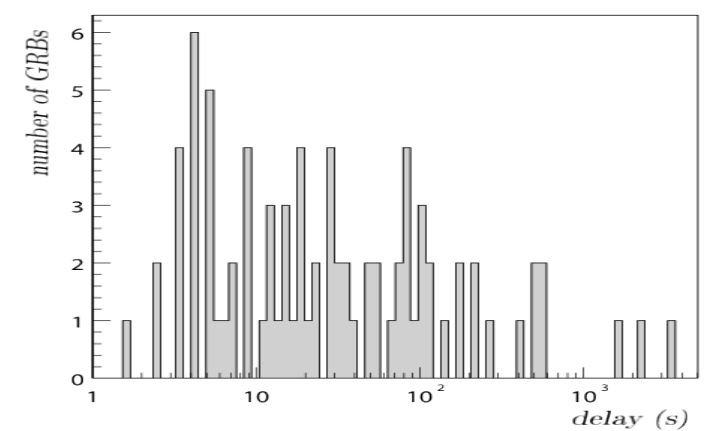

Figure 2: Distribution of the delay between the detection of a GRB by the satellite and the time of the alert message is distributed.

The ANTARES Data Acquisition (DAQ) system is designed around the all-data-to-shore concept, which entails the transport of all photon signals recorded by the optical modules to the shore station where filtering is performed. The filtering algorithms are operating in coincidence with a GRB alert, but in this case also raw data are saved on disks. A couple of minutes of unfiltered data (L0 data) buffered before the alert message are also available to be stored. This configuration is maintained for a couple of minutes, in fact even long GRBs have a typical duration typical duration less than two minutes. The filtered data are known as L1 data. A L1 hit is defined either as a local coincidence on the same storey (ANTARES consists of 295 storey with 3 PMTs each) within 20 ns, or as a single L0 hit with a large amplitude, typically 3 photoelectrons. When a muon event is triggered, all PMT pulses are recorded over $4 \mu \mathrm{s}$ in a snapshot. On the other hand the L0 data sample contains every signal detected above the 0.3 photoelectrons threshold for the whole alert duration (i.e. couple of minutes). 


\section{Analysis principle}

All the filtering on the L0 data is performed offline when the data sample is analysed and the position of the GRB is known with the best possible accuracy. A dedicated filtering algorithm has been developed for this data sample. In the case of the GRB, the direction of the potential neutrino events is known, so the algorithm can look for space-time correlations with a less strict filter condition because only one direction is considered. The direction of the muons that originate from GRB neutrinos is on average comparable with the direction of the burst, the angular spread depending on the neutrino energy. Using this algorithm more events are expected to be detected in the interesting direction, which would be lost using the standard ANTARES filtering system, which is more stringent and looks for correlations in all the directions of the sky.

The hits that satisfy the filtering condition are used for the reconstruction of the track direction. In this analysis we will use a special reconstruction algorithm (known as GridFit [12]) that is optimized for low energy (below $10^{3} \mathrm{GeV}$ ). As input all L0-hits (raw data) are taken and three hit selections (with different criteria) are performed. After the hits collection three reconstruction steps are implemented, the final one is based on a likelihood maximisation method. The reconstruction algorithm provides the direction of the neutrino and other useful parameters like the reconstruction quality $\mathrm{X}$, which will be used later in the optimization of track selection criteria. The parameter $\mathrm{X}$ is defined as $X=$ nhits $-1.1 \cdot r \log L$ where nhits is the number of hits used in the reconstruction and $\mathrm{rLogL}$ is the reduced $\log$-likelihood of the track hypothesis.

Using raw data, the special filtering algorithm, the optimized reconstruction algorithm and applying the search method developed in [7] we obtained a larger sensitivity for this analysis at lower energy with respect to the standard analysis. In this analysis the number of triggered events (before quality cuts) has been doubled for energies above $10^{5} \mathrm{GeV}$ with respect to the standard ANTARES analysis. At lower energy the increase is more significant up to a factor 5 at energies around $50 \mathrm{GeV}$.

A simulation of a neutrino flux has been performed with Genhen [13] with an energy spectrum rescaled according to photospheric model predictions. We assumed a simplified neutrino spectrum with a flux $\left(E_{v}^{2} d N / d E_{v} d t\right)$ of $5 \cdot 10^{-4} \mathrm{GeV} \mathrm{cm}^{-2} \mathrm{~s}^{-1}$ with cut-offs at $2 \cdot 10^{3} \mathrm{GeV}$ and $8 \cdot 10^{5} \mathrm{GeV}$.

Using this simulation we derived the point spread function of the reconstructed neutrino according to the GRB photospheric model. This function is used for building the signal probability density function (PDF), this function is called $S(\alpha)$, where $\alpha$ is the angle between the reconstructed track direction and the true MC neutrino direction in degree. The background $\operatorname{PDF} B(\alpha)$ is assumed uniform in the search window (10 degrees). Background events come from atmospheric neutrinos and from atmospheric muons which were misreconstructed as upwards going. In [7], the sum of these two is estimated from data. The small duration of runs in our case ( 2 mins instead of a few hours) and the higher dependence on biolumination conditions makes the direct application of this strategy impossible, in fact statistics prevents a solid direct estimate for a single 2-minute data sample. To overcome this we have simulated a small sub-sample of raw data (a tenth of runs distributed in ANTARES life, run duration is around 2 hours) and looked for a relationship between them and the corresponding sample of official ANTARES Run by Run (RbR) Monte Carlo simulation which takes in account the experimental conditions of each data run, such as the status of each PMT, the detector configuration, the actual environmental conditions and optical background [14]. The rate 
of upgoing muons can be evaluated introducing a ratio $\mathrm{C}$ as follows

$$
C=\frac{\frac{\mu_{\mu(\text { data }}(\text { data })}{\mu_{\uparrow}(M C)}}{\frac{\mu_{\downarrow}(M C)}{\mu_{\downarrow}(M)}}
$$

The ratio $\mathrm{C}$ is constant in time and weakly depends on the detector condition or bioluminescence background, so it can be used to evaluate the number of expected upgoing muons as

$$
\mu_{\uparrow}(\text { data })=C \cdot \mu_{\downarrow}(\text { data }) \frac{\mu_{\uparrow}(M C)}{\mu_{\downarrow}(M C)},
$$

where $\mu_{\uparrow} / \downarrow$ (data) is the number of upgoing/downgoing muons in a raw data files and $\mu_{\uparrow} / \downarrow_{\downarrow}(M C)$ is the number of upgoing/downgoing muons in the corresponding Run by Run Monte Carlo simulation. The random background due to random coincidences has been also simulated, but it is irrelevant compared to the muon background. It will be neglected in our background estimation.

The muon background estimation has been verified using a reduced number of raw data files that are associated to false GRB alarm. The estimation is compatible with the data especially for tracks of good quality. We also checked the dependence of this background estimation on the zenith of the event. The ratio rawdata/RbRMC does not change dramatically considering different zenith angle of our search window. In order to be more conservative an additional safety factor 2 is added to our muon background estimation to take in account the zenith dependence of the muon background.

\section{Sensitivity study}

The sensitivity study will be performed on a generic GRB with neutrino flux $\left(E_{v}^{2} d N / d E_{v} d t\right)$ of $5 \cdot 10^{-4} \mathrm{GeV} \mathrm{cm}^{-2} \mathrm{~s}^{-1}$ and cut-offs at $2 \cdot 10^{3} \mathrm{GeV}$ and $8 \cdot 10^{5} \mathrm{GeV}$. The GRB is assumed to be located in the part of sky where the ANTARES visibility is maximal.

According to the signal and background PDFs, as previously defined, pseudo-experiments are produced to derive the distribution of the log-likelihood ratio Q, obtained by maximizing the loglikelihood for the signal and comparing with the background only value. The extended maximum likelihood Q is defined as

$$
Q=\max _{\mu_{\text {sig }}} \sum_{\text {event }}^{n_{\text {tot }}} \log \frac{\mu_{\text {sig }} \cdot S\left(\alpha_{i}\right)+\mu_{b g} \cdot B\left(\alpha_{i}\right)}{\mu_{b g} \cdot B\left(\alpha_{i}\right)}-\left(\mu_{\text {sig }}+\mu_{b g}\right),
$$

where $\mathrm{S}$ and $\mathrm{B}$ represent the signal and background PDF as previously defined, $\mathrm{i}$ is the index of the event with space angle $\alpha_{i}$ with respect to the GRBs direction, $\mu_{b g}$ is the expected number of background events and $\mu_{\text {sig }}$ is the signal contribution. In Fig. 3 the distribution of the log-likelihood ratio $\mathrm{Q}$ is shown.

Evaluating the different curves of Fig. 3, we can compute the model discovery potential (MDP) and the expected sensitivity as a function of the expected number of events. This strategy is repeated for several values for the cut on the quality parameter $\mathrm{X}$ in order to find the selection that maximizes the model discovery potential. 


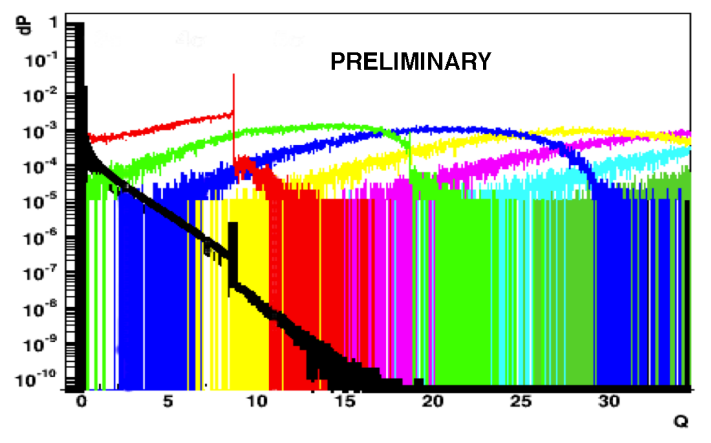

Figure 3: Log likelihood ratio $(\mathrm{Q})$ distributions. Background rate: 0.01. Yellow: background only. Red (green, blue, ...): background $+1,2,3, \ldots$ signals.

Using this optimal parameter we can derive the sensitivity as the $90 \%$ Confidence Limit that can be put on the flux considering the median background $\mathrm{Q}$ value. In the case of the spectrum considered in this analysis the sensitivity is $\left(E_{v}^{2} d N / d E_{v} d t\right) 3 \cdot 10^{-1} \mathrm{GeV} \mathrm{cm}^{-2} \mathrm{~s}^{-1}$.

We present in Fig.4 the expected efficiency improvement factor with respect to the same analysis applied on classical filtered data.

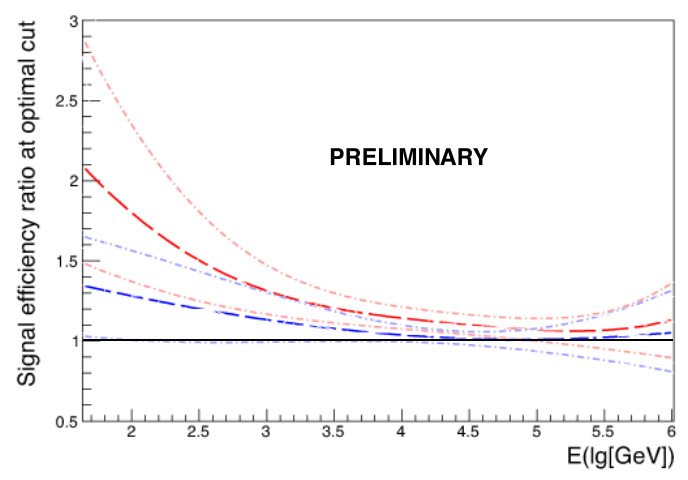

Figure 4: Signal efficiency ratio at optimal cut between the proposed analysis and the standard ANTARES analysis. Red: raw data filtered with the directional trigger and reconstructed with GridFit. Blu: standard data reconstructed with GridFit. Pale red and blu: corresponding 90\% confidence interval. All results are normalized to the standard ANTARES analysis.

As expected the proposed analysis has a better efficiency respect to the standard analysis at low energies, in particular the effectiveness is almost doubled at energies of a few hundred of GeV. Finally the sensitivity on the expected flux from GRB 130427A according to photospheric model [4] has been derived applying the same quality cut on the quality parameter as in the previous case (Fig.5).

\section{Conclusions}

Adapting the strategy used to search for neutrinos from GRBs based on the widely used inter- 


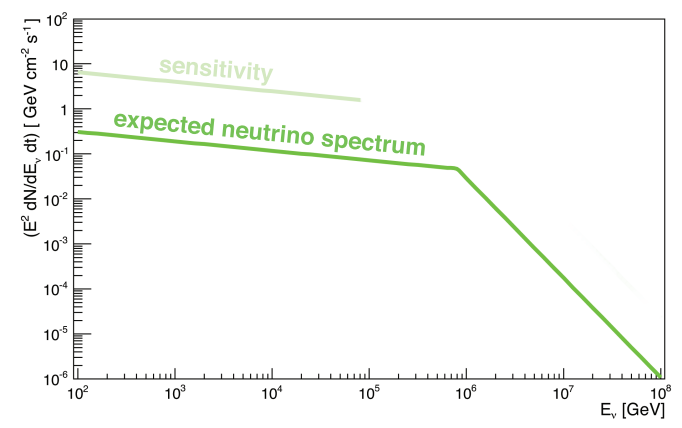

Figure 5: Expected neutrino spectrum (dark green) and sensitivity (pale green) according to photospheric model for GRB 130427A.

nal shock model, we have studied the sensitivity of ANTARES to the concurrent GRB photospheric model. This has been done using special ANTARES data and tools enhancing the sensitivity between $50 \mathrm{GeV}$ and $10 \mathrm{TeV}$. In order to enhance the sensitivity of the ANTARES in the range between $50 \mathrm{GeV}$ and $10 \mathrm{TeV}$ some dedicated tools have been used for this analysis: a special data sample of raw data, a directional trigger and a reconstruction algorithm optimized for this energy range. A solid way to estimate the upgoing muon has been found and the optimization of the selection criteria has been performed and a generic sensitivity obtained. This analysis will be applied on the data collected in correspondence of the two ANTARES best candidate for GRB detection of the last years (GRB110918A and GRB130427A).

\section{References}

[1] Paciesas W. S. et al. , Astrophys.J.Suppl. 122 (1999) 465-495

[2] Meegan et al. , Nature 355:143, 1992

[3] Gao S. et al., JCAP11 058, 2012

[4] Zhang B., Kumar P., Phys. Rev. Lett. 110, 121101, 2013

[5] Thompson C., Astrophysical Journal, vol. 664, 2007

[6] M.G. Aartsen et al, Astrophys.J. 1, 805 L5 (2015)

[7] S. Adrian-Martinez et al., A\&A, Volume 559, A9 (2013)

[8] S. Adrian-Martinez et al., JCAP03(2013)006

[9] Thompson C., M.N.R.A.S 270, 480, 1994

[10] D. Band et al., Astrophys.J. 413, 281 (1993).

[11] R. Abbasi et al, Nature 484, 351 (2012)

[12] E. Visser, Doctoral Thesis, Leiden University, 2015

[13] Brunner J., in VLVnT Workshop (Amsterdam), ed. E. de Wolf (Amsterdam:NIKHEF), http://www.vlvnt.nl/proceedings.pdf, 2003

[14] A. Margiotta for the ANTARES collaboration, Nucl.Instrum.Meth., A725, 98-101 (2013). 